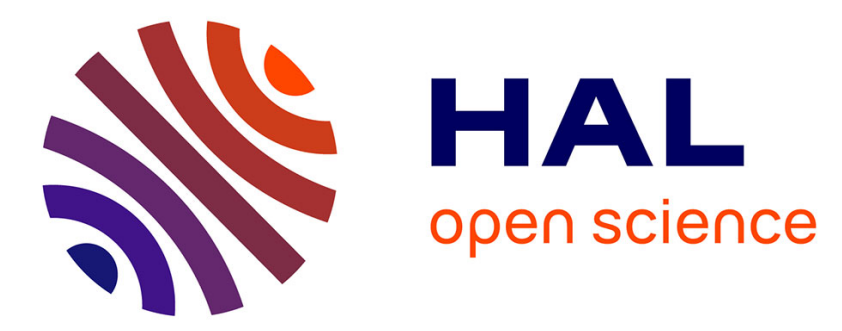

\title{
Supporting the Strategies Alignment Process in Collaborative Networks
}

\author{
Beatriz Andrés, Raul Poler
}

\section{To cite this version:}

Beatriz Andrés, Raul Poler. Supporting the Strategies Alignment Process in Collaborative Networks. 8th Doctoral Conference on Computing, Electrical and Industrial Systems (DoCEIS), May 2017, Costa de Caparica, Portugal. pp.3-19, 10.1007/978-3-319-56077-9_1 . hal-01629573

\section{HAL Id: hal-01629573 \\ https://hal.inria.fr/hal-01629573}

Submitted on 6 Nov 2017

HAL is a multi-disciplinary open access archive for the deposit and dissemination of scientific research documents, whether they are published or not. The documents may come from teaching and research institutions in France or abroad, or from public or private research centers.
L'archive ouverte pluridisciplinaire HAL, est destinée au dépôt et à la diffusion de documents scientifiques de niveau recherche, publiés ou non, émanant des établissements d'enseignement et de recherche français ou étrangers, des laboratoires publics ou privés. 


\title{
Supporting the Strategies Alignment Process in Collaborative Networks
}

\author{
Beatriz Andres and Raul Poler \\ Research Centre on Production Management and Engineering (CIGIP). \\ Universitat Politècnica de València (UPV). Calle Alarcón, 03801 Alcoy, Spain \\ \{bandres, rpoler\}@cigip.upv.es
}

\begin{abstract}
The establishment of collaborative relationships with the network partners provides them important advantages, such as competitiveness and agility, when responding to the current rapid market evolutions. Nonetheless, the participation in collaborative networks becomes a complex process that starts with the alignment of all the enterprises' objectives and strategies. Smart systems and approaches are needed in order support collaborative partners to deal with the strategies alignment challenge. The lack of alignment emerges because each enterprise defines its own objectives and strategies, to perform their business, and it could happen that non-compatible strategies are activated, involving the appearance of conflicts between strategies of different enterprises. To this regard, a decision support system is proposed, consisting of a mathematical model, a system dynamics method, a simulation tool and a guideline, with the main aim of supporting the process of identifying aligned strategies, among the enterprises of the collaborative network.
\end{abstract}

Keywords: alignment, strategy, objective collaborative network, performance indicator, system dynamics, simulation.

\section{Introduction}

The concept of collaborative networks $(\mathrm{CN})$ has been widely studied over the last years due to the positive effects undergone by the enterprises that collaborate[1]. In the work of consolidating a new discipline in $\mathrm{CN}$, Camarinha-Matos and Afsarmanesh [2] define $\mathrm{CN}$ as a network consisting of a variety of autonomous and heterogeneous entities that collaborate to better achieve common or compatible goals, to jointly generate value, and whose interactions are computer network supported. Collaborative processes have been widely studied over the last years due to their decisive contribution in the proper operation of the $\mathrm{CN}$. With the aim of consolidating the wealth of knowledge in the research area of collaborative processes Andres and Poler [3] perform a deep analysis that has allowed to (i) classify the most relevant collaborative processes according to the decision making level: strategic, tactical, and operational, and (ii) analyse for each process the models, guidelines and tools proposed in the literature to address them. The authors conclude that amongst all the collaborative processes studied, the ones that need to be addressed from the collaborative perspective, through proposing new contributions to fill the 
decentralized and collaborative features, are (i) at the strategic decision-making level: the strategies alignment process; (ii) at the tactical level: share costs and profits, and uncertainty management; and (iii) at the operational level: collaborative lotsizing.

Enterprises willing to collaborate must overcome a set of barriers not only associated with the establishment of collaborative processes identified by [3] [4] (e.g. products design, demand forecasting, operations planning, replenishment, uncertainty management, share costs and profits, scheduling, information exchange, interoperability, etc.), but also when defining compatible goals, activating complementary strategies [5] or aligning their core values [6][7]. Focusing on the strategies alignment process, the mere consideration of all the enterprises' objectives when deciding which strategies are the best ones to carry out will allow achieving higher levels of adaptability, agility, and competitiveness [1], strengths that are specially valued in current turbulent contexts and dynamic markets. Considering this, the strategies alignment process is hereafter addressed; with the main aim of dealing with the conflicts appearing when strategies misalignments emerge, in the $\mathrm{CN}$ context. Intuitively, as the activation of strategies has a direct influence on the objectives achievement, it can be understood that the strategies will be characterised by being aligned when each activated strategy not only promotes the achievement of the objectives defined by the enterprise that formulates such strategy, but also when positively influences the accomplishment of the objectives defined by the rest of the network partners.

Considering the importance of aligning strategies, among the enterprises of the same network, in terms of improving the $\mathrm{CN}$ relationships, there is a lack of an integrated approach to support enterprises on the modelling, assessment and solution of the strategies alignment process from a collaborative and integrated perspective. In the light of this, the following research questions are raised to support the strategies alignment process, in order to solve them as the objective of this research.

RQ1. How to model the impact that each strategy, formulated by one enterprise, has on the objectives defined by the other network enterprises? That is, how to model the impact of the strategies at the inter-enterprise level?

RQ2. What would be an adequate model to support the process of identification of aligned strategies, through modelling the strategies impact in the objectives, in $\mathrm{CN}$ context?

RQ3. What would be an adequate method to support the process of identification of aligned strategies, and to represent causal relationships (impacts) between the strategies and the objectives, in $\mathrm{CN}$ context?

RQ4. What would be an adequate tool to support the process of identification and assessment of aligned strategies, and to compute the strategies impact on the objectives performance at enterprise and network level, in $\mathrm{CN}$ context?

RQ5. What would be an adequate guideline to support the process of identification and assessment of aligned strategies, and to analyse the strategies impact on the objectives and identify misalignments, in $\mathrm{CN}$ context?

In order to give response to the raised research questions, the paper is organized as follows: Section 2 discusses the relationship between the approach proposed in this paper, to deal with the strategies alignment process, and the Smart Systems; Section 3 summarizes literature review performed in the research area of strategies alignment; Section 4 introduces the decision support system, consisting of a mathematical model, 
a system dynamics method, a simulation tool and a guideline, with the main aim of supporting the process of identifying aligned strategies among the enterprises of the $\mathrm{CN}$; Section 5 the proposed approach is validated in a real use case from food industry; finally, Section 6 provides the conclusions and the paper discussion.

\section{Relationship with Smart Systems}

Smart Systems (SS) have multi-disciplinary applications in different areas of research, such as the social, economic, healthcare, energy, safety and security, logistics, ICT, and manufacturing. The area under study of this paper is focused on the $\mathrm{CN}$ operation, which includes service and manufacturing sectors. The novelty in SS is the integration of different components, regardless the technologies and materials in which are created [29]. The proposed strategies alignment approach is applied in different industries and sectors that have in common the $\mathrm{CN}$ to which they belong; in this regard, SS can support the diversity associated to this approach. The decision support system proposed could be used as part of a SS that allows, through using real time information of other interoperable components, identifying in each enterprise the most appropriate aligned business strategies. The negotiation process, for identifying the most appropriate strategies, could be also included in a SS in order to allow enterprises make smart decisions with regards the strategies to activate during their participation in the $\mathrm{CN}$. In the light of this, the strategies alignment approach proposed could benefit from the real-time information, response capability, tracking and monitoring features that provide the SS. The integration of different systems, for the implementation of the strategies alignment approach in SS, is a key question to answer, bringing together interdisciplinary technological approaches and solutions for overcoming potential limitations in the establishment of collaborative process and the stable and sustainable operation of the $\mathrm{CN}$.

\section{Problem Definition and Conceptualization}

The literature review carried out in [3] has allowed identifying, firstly, the most important processes to perform in a $\mathrm{CN}$, and secondly, amongst all these processes, those that have a lack of contributions from the CN context. As stated in Section 1, the strategies alignment process is included in the group of potential processes to propose solutions in collaborative decentralised scenarios. According to the analysis carried out in [3] it can be concluded that, to the best of our knowledge, the strategies alignment process is a collaborative process that requires to be studied, so that models, guidelines and tools for its analysis, assessment and resolution, in the $\mathrm{CN}$ context, have to be proposed.

In a network of enterprises, the alignment can be defined as a proper or desirable coordination or relationship of the components of this network. More concretely, in the management field, the concept of alignment can be considered as a situation in which the strategies, formulated by the entities belonging to the network, are strictly combined under a set of functions to achieve the objectives [8]. CNs consist of 
autonomous and heterogeneous enterprises [9] each one defining its own objectives. The formulation of the strategies answers the question: How to reach the objectives? Once the planner decides the scope, situation or problem that aims to modify, a goal is drawn to guide the processes of change and then to trace the trajectory of necessary events over time to achieve that purpose. Strategy is the way forward to achieve the objectives. The business strategies are the set of actions raised to achieve the defined objectives; therefore, each enterprise of the $\mathrm{CN}$ formulates its own strategies with the main aim of achieving the defined objectives. There will be times in which all the strategies formulated are activated. Nevertheless, sometimes only a few of the formulated strategies will be activated, due to, for example, a restriction associated with the budged. Lets consider two enterprises (E) in a $\mathrm{CN}$, each one defines two objectives $(O)$ and formulates two strategies $(s t r)$. Each objective has associated a KPI to measure its achievement. In this regard, $E_{1}$ acquires the role of the distributor in the $\mathrm{CN}$ and defines $O_{11}$ : Increase the product sales by a $10 \%$, and $O_{12}$ : Reduce the product costs by a 30\%; and formulates str ${ }_{11}$ : Invest 0,5 m.u on marketing activities, and str $_{12}$ : Conduct negotiations with other manufacturers to reduce the purchasing costs. $\mathrm{E}_{2}$ acquires the role of the manufacturer in the $\mathrm{CN}$ and defines $O_{21}$ : Increase the profit by a $15 \%$, and $O_{22}$ : Reduce the quantity of product that cannot be sold by 100 $\%$; and formulates str $_{21}$ : Use different distribution channels to sell the product in other markets, and str $_{22}$ : Buy one machine to make derivative products, reprocessing the product that cannot be sold (i.e. low cost product). With this example it can be observed that the $s t r_{12}$ is not compatible with the $s t r_{21}$, because $s t r_{12}$ is devoted to establish new relations with other manufacturers, which will involve the reduction of the profit defined in $O_{21}$. Moreover, if $E_{1}$ conducts negotiations with other manufacturers $\left(s t r_{12}\right)$, the $O_{22}$ will be negatively influenced. Focusing on $E_{2}$, the $s t r_{21}$ focuses on the generation of alliances with distribution channels different from the one provided by $E_{1}$. The activation of $s r_{21}$ negatively influences $O_{11}$, defined for reducing the product sales; and consequently the $O_{12}$ that leads to reduce the product costs. Considering the aforementioned, the $s_{12}$ and $s r_{21}$ are considered misaligned, if activated at the same time. On the other hand, $s r_{11}$ and $s r_{22}$ are considered to be aligned because the two formulated strategies positively influence the achievement of the objectives defined. Assuming that, the strategies alignment concept is defined next as: "the set of strategies, formulated by the enterprises belonging to the $\mathrm{CN}$, whose activation positively influence, on the whole, the objectives achievement of the majority of the enterprises participating in the $\mathrm{CN}$; obtaining the best performance at the network level, although small number of the strategies negatively influence any of the defined objectives" [10]. It must be considered that (i) individual enterprises take part in several networks, so that it is likely that some of the enterprises taking part in these networks have contradictory objectives and consequently contradictory strategies; and (ii) the enterprises belonging to one specific $\mathrm{CN}$ are heterogeneous and contradictory objectives and strategies might arise. Therefore, for enterprises belonging to a $\mathrm{CN}$, the defined objectives and the strategies formulated by one enterprise could favour, or not, the objectives defined by other enterprises. In order to achieve the ideal situation, enterprises belonging to a $\mathrm{CN}$ should be able to identify those aligned strategies, whose activation promotes the improvement of the objectives defined by the majority of the networked enterprises, or at least the activated strategies do not negatively influence on the objectives attainment [5]. 


\section{Literature Review}

A summary of the review performed to analyse how the strategies alignment process has been treated in the literature is presented. In the light of this, some models guidelines and tools are identified and briefly described. The gaps and trends related to the strategies alignment process from a collaborative perspective are identified, as a result of the analysis performed. The initial round of search was based on a broad meaning of keywords and contexts (enterprise and network level), to ensure that papers adopting an alternative nomenclature, were identified. Alignment of strategies, alignment of actions, alignment of decisions, collaborative decisions design, collective decisions and alignment in supply chain where the keywords used. The found works proposed models, guidelines and tools to deal with the alignment of decisions from different decision making levels and different perspectives of application (i) one in which the decisions are collaboratively made and from the beginning of the decision making the decisions are aligned, and (ii) another one in which the each partner defines its own decisions and then these are pooled in order to identify those that are more aligned with the decisions of other network partners. Considering the reviewed works, Table 1 is generated, listing and briefly describing the works. Due to space restrictions the table presents works from 2012. The selected contributions are analysed considering if the proposed approaches are designed when (i) the decisions are collaboratively and centralised (C) made or, unlike, (ii) the decisions are decentralised (D) made by each $\mathrm{CN}$ partner and after that the decisions are aligned. A set of models, guidelines and tools are proposed in the literature with the main aim of aligning decisions among the enterprises of the network. Some of them can be highlighted: classified as models it can be found the multi-criteria methods such as FMCDS or MCDM; fuzzy approaches deal with uncertain information. As regards the guidelines, collaborative strategies or negotiation-based schemes such as S-DSP are found. Considering the methods, TOPSIS, MCOGA, GA, ANP, causal maps can be emphasised. Concerning tools MECDSS is found. Despite of the importance of aligning strategies, in terms of avoiding partnership conflicts, to the best of our knowledge, there are some gaps in the literature as regards contributions that provide a holistic approach that allows considering all the strategies formulated by all the partners in the $\mathrm{CN}$ context. The performed review has allowed identifying possible trends, gaps and actions in the topic under study. This actions are summarised as follows: (i) propose a complete approach to deal with the strategies alignment process by considering all the strategies formulated by all the enterprises of the $\mathrm{CN}$; (ii) identify the aligned strategies from an holistic perspective regardless of their nature and type, taking into account the $\mathrm{CN}$ context; (iii) model the strategies alignment process considering the intra-enterprise strategies alignment (alignment of the strategies defined in the same enterprise), and inter-enterprise strategies alignment (alignment among the strategies defined by different enterprises of the network); (iv) consider the performance approach to measure the strategies influence, that when activated, it will be measured considering the increase and decrease of the KPIs defined in each enterprise. Definitely, from the performed literature review, there is a need to propose a framework consisting of a model method, tools and guidelines to address the strategies alignment process from a holistic perspective by equally considering all the network partners. 
Table 1. Contributions dealing with the research topic decisions alignment

\begin{tabular}{|c|c|c|c|}
\hline Author & Proposal & C & D \\
\hline [11] & $\begin{array}{l}\text { An optimisation model to configure supply chain network, by combining optimisation at the } \\
\text { strategic and tactical level }\end{array}$ & $\mathrm{X}$ & - \\
\hline [12] & $\begin{array}{l}\text { A set of decisions are previously identified and with IMGP (Interactive Meta-Goal } \\
\text { Programming) approach is used to analyse a decision under consideration and identify how it } \\
\text { favours other enterprises performance }\end{array}$ & - & $\mathrm{x}$ \\
\hline [13] & $\begin{array}{l}\text { Sustainability - Decision Support Protocol (S-DSP) is a collaborative centralised solution that } \\
\text { enables to maximise the sustainability of supply network in production and scheduling } \\
\text { planning }\end{array}$ & $\mathrm{X}$ & - \\
\hline [14] & $\begin{array}{l}\text { Multi-enterprise collaborative decision support system (MECDSS) that allows each enterprise } \\
\text { to propose its own decisions and dispose of all the data as regards the decision-making } \\
\text { components of its partners. Enterprises identify how its decisions affect itself and its partners }\end{array}$ & - & $\mathrm{x}$ \\
\hline [15] & $\begin{array}{l}\text { A multi-criteria decision making (MCDM) technique, is proposed to support decision making } \\
\text { in B 2B collaboration }\end{array}$ & $\mathrm{X}$ & - \\
\hline [16] & $\begin{array}{l}\text { Analytic Network Process (ANP) used to determine how the decisions defined by the CN } \\
\text { enterprises influence on the strategies achievement of the network }\end{array}$ & - & $\mathrm{X}$ \\
\hline [17] & $\begin{array}{l}\text { Fuzzy analytic hierarchy process (FAHP) that uses weightings to support the identificaiton of } \\
\text { the importance of the dimensions that influence the decisions }\end{array}$ & $\mathrm{X}$ & - \\
\hline [18] & $\begin{array}{l}\text { Fuzzy causal maps and qualitative assessment methods designed to asses the values alignment } \\
\text { among the CN partners }\end{array}$ & - & $\mathrm{X}$ \\
\hline [19] & $\begin{array}{l}\text { Fuzzy TOPSIS (Technique for order preference by similarity to ideal solution) and multi- } \\
\text { criteria decision making (MCDM) are used to make collective decisions to solve a number of } \\
\text { problems, which are characterised by various quantitative and qualitative criteria }\end{array}$ & $\mathrm{X}$ & - \\
\hline [20] & $\begin{array}{l}\text { TOPSIS combined with a multi-criteria genetic optimisation feature. Modified multi-criteria } \\
\text { genetic optimisation feature (MCOGA) are proposed to create centralised collaborative } \\
\text { decision }\end{array}$ & $\mathrm{X}$ & - \\
\hline [21] & $\begin{array}{l}\text { Optimisation model to integrate the decision of partners' selection and collaborative } \\
\text { transportation scheduling. A novel Genetic Algorithm }(\mathrm{GA}) \text { is proposed }\end{array}$ & $\mathrm{X}$ & - \\
\hline [22] & $\begin{array}{l}\text { An approach based on subjective probabilities is proposed to evaluate (i) the probability that a } \\
\text { decision of one partner is optimal for itself and (ii) the probability that a decision for the first } \\
\text { partner is optimal for other partners }\end{array}$ & - & $\mathrm{X}$ \\
\hline
\end{tabular}

\section{Approach to Support the Strategies Alignment Process}

An approach that consists of a model, method, tool and guideline is proposed, to deal with the strategies alignment process, in the $\mathrm{CN}$ context.

\subsection{Mathematical Model}

The proposed model allows to formally represent, in a mathematical notation, the influences that the strategies activated in one enterprise have on the performance indicators (KPI) defined to measure the achievement of the objectives, both in the same enterprise and in other $\mathrm{CN}$ enterprises [10]. In order to represent the influences and relations between the KPIs and the strategies a mathematical notation model is proposed: the Strategies Alignment Model (SAM). First of all, the set of parameters and decision variables, used to model the SAM, are defined in Table 2. 
Table 2. Index and model parameters

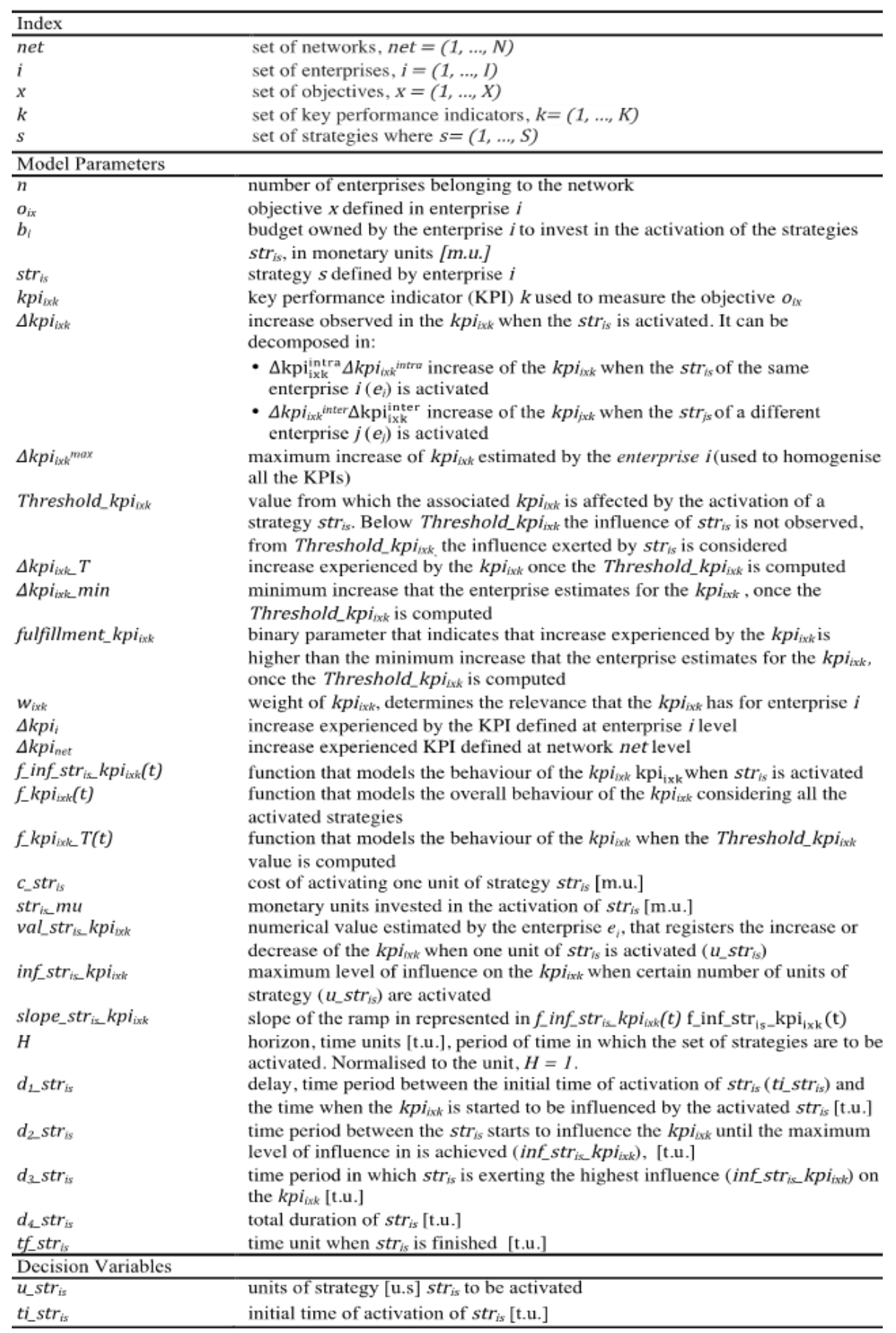


The SAM is hereafter developed, consisting of an objective function and the associated restrictions, representing the relations amongst all the defined variables and parameters. The main aim is to identify, amongst all the strategies defined, those strategies that have higher level of alignment. The activation of the aligned strategies positively influences the majority of the objectives defined by the networked partners, maximising the performance at network level. The SAM computes the KPIs improvement or worsening when a strategy is activated. Thus, the developed model supports enterprises on the decision making as regards the number of units of strategy $\left(u_{-} s t r_{i s}\right)$ to be activated and the time in which the strategies have to be activated ( $t i_{-} s t$ ris $_{i s}$ ) with the objective of maximising the network performance, given by $k p i_{\text {net }}^{s}$ as the homogenised version of the kpi $_{\text {net }}$. Therefore, the objective function of the SAM is mathematically represented by the following equation (1):

$$
\max \text { Akpi net }
$$

The homogenised version of $\Delta k p i_{\text {net }}$ is obtained through the homogenisation of parameters related to KPIs ( $\left.\Delta k p i_{i x k}^{n}\right)(2)$; and the normalisation of the parameters related to durations and time (3), based on the horizon $(H)$ of time in which the strategies alignment process is modelled.

$$
\begin{aligned}
& \Delta k p i_{i x k}^{b}=\frac{\Delta k p i_{i x k}}{\Delta k p i_{i x k}^{i m a x}}
\end{aligned}
$$

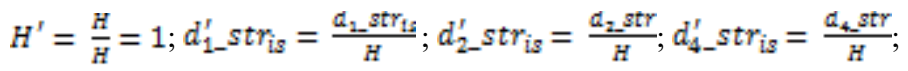

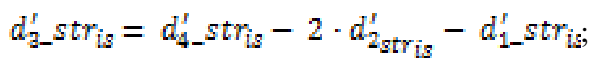

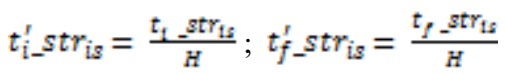

Two decision variables, $u_{-} s_{\text {st }}$ is and $t$ stris, are defined in order to maximise the parameter $\Delta k p i_{\text {net }}^{*}$. The decision variable $u_{-} s t r_{i s}$ decomposes the strategy $\left(s t r_{i s}\right)$ in units of strategy, allowing representing the "intensity" in which each strategy stris is activated. One unit of strategy has an associated a cost $\left(c_{-} s t r_{i s}\right)$. Therefore, depending on the parameter $c_{-} s t r_{i s}$, the enterprise' budget $\left(b_{i}\right)$ will be reduced in a lesser or larger extent (4). The budget, $b_{i}$, owned by each company defines the monetary capacity constraint (5). In order to identify the influence that one unit of strategy $\left(u_{-} s t r_{i s}=1\right)$ has over the $\Delta k p i_{i x k}^{n}$, parameter val_str ${ }_{i s-} k p i_{i x k}^{n}$ is used (6).

$$
s t r_{i \mathcal{i}} m u=u_{-} s t r_{i s}-c_{-} s t r_{i s}
$$




$$
\begin{aligned}
& b_{\mathrm{i}} \geq \sum_{s} s t r_{\mathrm{i} s-m u} \quad \forall s
\end{aligned}
$$

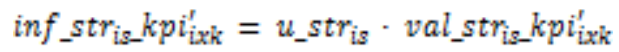

The influence that one strategy $s t r_{i s}$ has on a particular $\Delta k p i_{L x k}^{i}$ is modelled through the function $f_{-} i n f_{-} s t r_{i g-} k p i_{i x k}^{t}$. This function, $f_{-} i n f_{-} s t r_{i g-} k p i_{i x k}^{0}(8)$, is a piecewise function that depends on the time $\left[f_{1}(t)\right]$, that is, the duration parameters ( $d_{1} \_s t r_{i s}, d_{2} s t_{i s}, d_{3} s t_{i s}$ and $d_{4} s t_{i s}$ ) and the decision variable ti_stris. Besides,

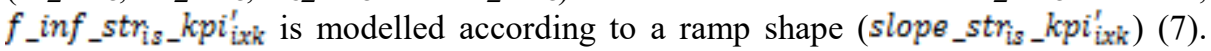
The representation of the ramp allows modelling that, after the delay time ( $\left.d_{1} \_s t r_{i s}\right)$, the stris $_{\text {is }}$ progressively influences the $k p i_{L X K}^{a}$.

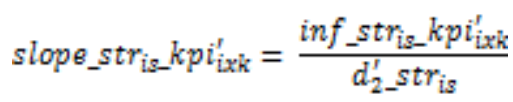

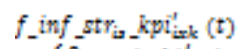

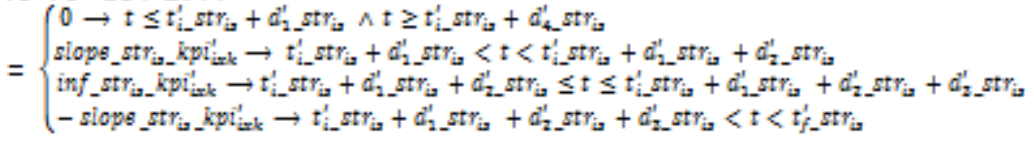

The influence received by the KPIs defined in one enterprise $i(11)$ is caused by both intra-enterprise influence, $\Delta^{\text {intra }} k p_{i x k^{x}}^{g}$ (9) and inter-enterprise influences, $\Delta^{\text {inter }} k p i_{i x k}^{i n},(10)$.

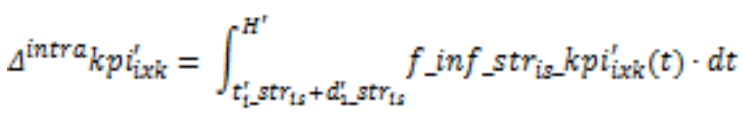

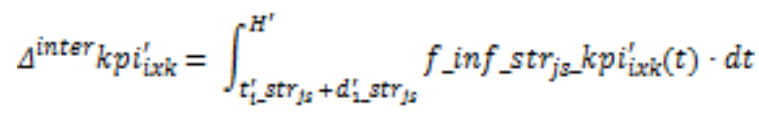

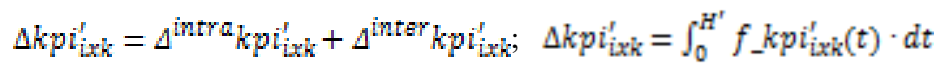


After being depicted the function $f_{-} k p i_{i x k}^{i}$ and computed the $\Delta k p i_{i x k}^{i}$, the value estimated by the threshold (Threshold_kpi $i_{i x k}^{b}$ ) must be considered (12). At enterprise and network level the parameters $\Delta k p i_{i}^{b}$ and $\Delta k p i_{\text {net }}^{s}$ are defined as (13).

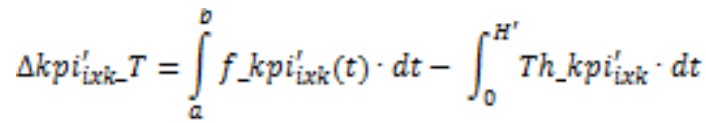

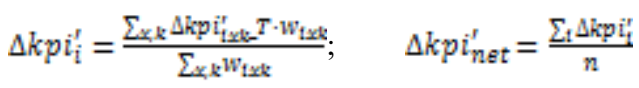

\subsection{System Dynamics Method}

The method used is based on system dynamics (SD), and will allow to graphically represent and solve the proposed mathematical model, from a CN perspective. SD will enable to characterise the causal relationships between the strategies and the objectives; modelling the influences that the objectives experience when certain set of strategies are activated. Moreover, SD will favour to understand the structure and dynamics of complex systems, such as the CN [10] [23]. The causal loop diagram is the graphical description that represents the system in SD. It includes all the system elements and represents the relationships among them. The causal diagram allows to qualitatively represent the behaviour of the modelled system. In order to carry out a quantitative analysis the flow diagram is constructed. The flow diagram interprets the causal loop diagram (the information and the casual relationships depicted) into a terminology that allows transcribing the equations within a simulation software. The parameters modelled in the SAM are translated for its use in the SD simulation software. Moreover the equations remain as shown in Table 3. The flow diagram of the SD SAM is represented in Figure 1, and will be extended according to the number of enterprises, the number of KPIs defined and the strategies formulated. 


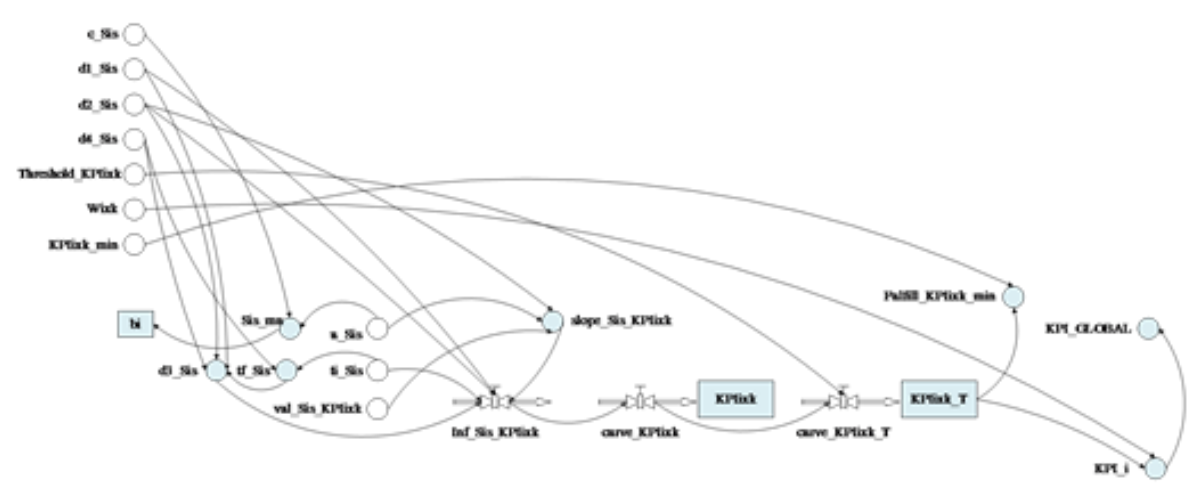

Fig. 1. Flow diagram in SD of the Strategies Alignment Model.

Table 3. Equations of the flow diagram

\begin{tabular}{|c|}
\hline $\begin{array}{l}\text { dimension_KPIixk, representing the indexes of the KPIs defined in the model index_KPIixk } \\
\text { dimension_Sis, representing the indexes of the strategies defined in the model index_Sis }\end{array}$ \\
\hline $\mathrm{bi}-\Sigma$ Sis mu \\
\hline Sis_mu $=$ u_Sis $\cdot$ c_Sis.get (index_Sis) \\
\hline tf $\mathrm{Sis}=\mathrm{ti}$ Sis $+\bar{d} 4$ Sis.get (index Sis) \\
\hline $\begin{array}{l}\text { d3_Sis }=\text { d4_Sis.get(index_Sis) - d1_Sis.get(index_Sis) - } \\
(2 \cdot \text { d2_Sis.get(index_Sis)) }\end{array}$ \\
\hline $\begin{array}{l}\text { slope_Sis_KPIixk }=(\text { u_Sis } \cdot \text { val_Sis_KPIixk [dimension_KPIixk])/ } \\
\text { d2_Sis.get(index_Sis) }\end{array}$ \\
\hline $\begin{array}{l}\text { Inf_Sis_KPIixk = delay (ramp (slope_Sis_KPIixk[dimension_KPIixk], } \\
\text { ti_Sis, ti_Sis + d2_Sis.get(index_Sis)) - ramp } \\
\text { (sIope_Sis_KPIixk[dimension_KPIixk], ti_Sis + d2_Sis.get(index_Sis) + } \\
\text { d3_Sis, ti_Sis +2.dd2_Sis.get(index_Sis) + d3_Sis), } \\
\text { d1_Sis.get(index_Sis)) }\end{array}$ \\
\hline curve_KPIixk $=\sum \operatorname{Inf}$ S11_KPIixk[dimension_KPIixk] \\
\hline KPIixk $=\int$ curve_KPIixk[dimension_KPIixk] \\
\hline $\begin{array}{l}\text { Curve_KPIixk_T }=\overline{\text { IF }}((\text { curve_KPIixk[dimension_KPIixk] }>= \\
\text { Threshold_KPIixk[dimension_KPIixk]) THEN (curve_KPIixk[dimension_KPIixk] } \\
- \text { ThreshoId_KPIixk[dimension_KPIixk]) ELSE (IF } \\
\text { (curve_KPIixk[dimension_KPIixk]<0) THEN curve_KPIixk[dimension_KPIixk] } \\
\text { ELSE 0)) }\end{array}$ \\
\hline KPIixk_T $=\int$ curve_KPIixk_T[ dimension_KPIixk ] \\
\hline $\begin{array}{l}\text { fulfill_KPIixk_min }=\text { IF }((\text { KPIixk T[ dimension_KPIixk ] }>=\text { KPIixk_min[ } \\
\text { dimension_KPIixk ] }) \text { THEN } 1 \text { ELSE } 0)\end{array}$ \\
\hline KPI_i $=\sum$ KPIixk_T.get (index_KPixk) $\cdot$ Wixk[dimension_KPIixk] \\
\hline KPI_GLOBAL $=\Sigma \mathrm{KPI} i \mathrm{i} / \mathrm{n}$ \\
\hline
\end{tabular}

\subsection{Simulation Tool}

The proposed simulation software tool is used to solve and represent the strategies alignment model, based on SD rigorous method. The use of computational tools 
allows automatically solving the strategies alignment process. System-dynamic's simulation based models supports on the process of computing the strategies to activate and the time slot in which activate them, optimising the global performance of the CN. Considering the SAM developed and the SD resolution method described, three tools used to address the strategies alignment process, from a $\mathrm{CN}$ context, are described: (i) AnyLogic simulation software is selected to support the system dynamics (SD) method, in which the SAM is solved; (ii) a Database Management System (DMS) is proposed to store all the information required in the SAM. The parameters required to feed the SAM are gathered in a Microsoft Access Database specifically designed; and (iii) the Strategies Alignment GENerator (SAGEN) is designed as an application to automatically generate the SAM in SD simulation software. In this regard, SAGEN contains the set of procedures that allow generating the required structure, in XML language, to create the strategies alignment simulation model in the SD simulation software selected (AnyLogic). The procedures are created according to the requirements of the XML schema, for its reading in AnyLogic. The programing language used to build SAGEN is Pascal. Lazarus [24] is used as an Integrated Development Environment (IDE) that uses Free Pascal compiler. In order to have a deeper insight of SAGEN programming and the procedures creation, we refer readers to [25]. To automatically generate the SAM in SD simulation software, the user firstly introduces the information required to solve the SAM in the DMS, through SAGEN user interface (SAGEN UI) (see Figure 2). SAGEN UI is connected with Microsoft Access Database 2010 through an OCDBConnection. The information stored in Microsoft Access Database 2010 contains all the tables and fields necessary to create the XML file that contains the SAM to be simulated in AnyLogic simulation software. In a second step, the user creates the XML file, which results from the execution of the procedures programmed in SAGEN. The XML file automatically created in SAGEN contains the strategies alignment simulation model, which can be loaded in AnyLogic simulation software. The SAM is automatically created containing the flow diagram, as well as the simulation and the optimisation experiments. AnyLogic simulation software is selected due to brings together the most common modelling methods: System Dynamics (SD), Discrete Events (DE), and Agent Based (AB). AnyLogic integrates both simulation and optimisation experiments. Accordingly, in the optimization experiments, AnyLogic searches the values of the model parameters that lead to obtain greater performance levels of the model, given an objective function and the set constraints and requirements. OptQuest is the engine used by AnyLogic to carry out the optimisation of the represented simulation model [26].

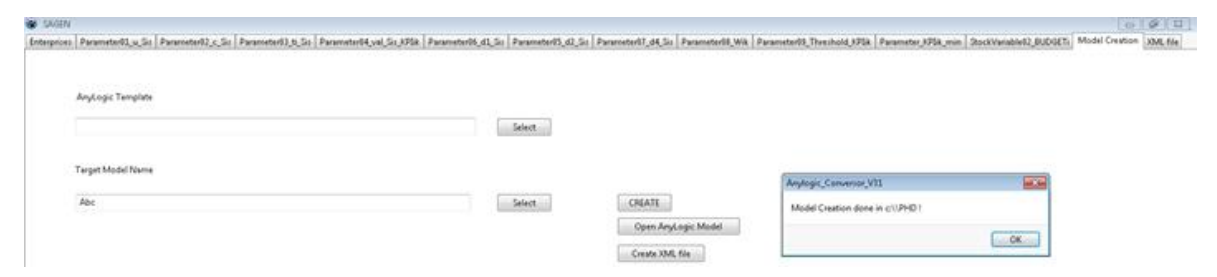

Fig. 2. SAGEN User Interface 


\subsection{Guideline}

A guideline is proposed as a complementary mechanism to the model, method and tool, with the main aim of supporting the enterprises, which belong to a $\mathrm{CN}$, on addressing, assessing and solving the strategies alignment process [27]. The guideline consists of twelve phases, hereafter briefly described. Phase 1 starts with the identification of the $\mathrm{CN}$ partners, willing to align their strategies. Phase 2 focuses on the enterprises' roles definition. Phase 3 continues with the collection of the data required as an input of the SAM related with the KPIs and the parameters associated $\left(k p i_{i x k}, \Delta k p i_{i x k}, \Delta k p i_{i x k}\right.$ max ,Threshold_kpi $\left.i_{i x k}, w_{i x k}\right)$. Phase 4 is devoted to the collection of data, from the $\mathrm{CN}$ enterprises, related with the strategies and the parameters associated (stris, $\left.c_{-} s t r_{i s}, d_{1} \_s t r_{i s}, d_{2} s t r_{i s}, d_{4} s t r_{i s}\right)$. In Phase 5 the collaborative partners agree the type of collaboration to carry out in the CN. Three collaboration levels (CL) are defined depending on the data exchanged: (i) CL1, enterprises only exchange information as regards the KPIs defined and enumerated $k p i_{i k}$; (ii) CL2, enterprises exchange information about the KPIs and the parameters that characterize them, and the number of strategies (only the IDs of the strategies, not the definition) and the parameters that characterize them; and (iii) CL3, enterprises exchange information as regards the KPIs defined and the parameters that characterize them, and the definition of the strategies formulated and the parameters that characterize them. In Phase 6, the values of influence are estimated by each enterprise, val str $r_{i s} k p i_{i x k}$. The data retrieved in Phase 4, 5 and 6 is gathered in Phase 7, by using a template. In Phase 8 the gathered data is introduced in the DMS. SAGEM allows automatically creating, in Phase 9, the SAM in the simulation software selected, AnyLogic. The resolution of the model is performed in Phase 10, and the SAM solutions are generated. The negotiation of the SAM results is performed in Phase 11, which depends on the collaboration type previously agreed. When negotiating, each enterprise selects the alternative of solution, that best fits to its requirements. The alternative of solution is exchanged with the other partners of the $\mathrm{CN}$, and a negotiation process is started until the CN partners agree on the alternative of solution selected, which generates the closest performance to the optimum for each partner. In order to give the reader a better insight of the negotiation process, a scheme of the Negotiation Process for the Level 1 of Collaboration is described in [27]. Finally, Phase 12 allows, after carrying out the negotiation, identifying potential appearing conflicts when activating certain strategies. In Phase 12, possible misalignments and negative-influences appearing in the alternative of solution selected are to be identified, analysed and solved.

\section{Validation of the Proposal}

The stage of verification and validation aim to assess, give credibility and accredit the proposed original work [28]. In order to show the relevance of the model, method, tool and guideline proposed to deal with the strategies alignment problem a three validation elements are considered: (i) validation of the research by peer reviewed publications; (ii) development of empirical experiments; and (iii) real application of the complete approach in two networks belonging to the food (Pilot 1) and automotive 
industry (Pilot 2). The implementation of the proposed contribution allows identifying critical points of application; and the pilots allow showing the use that the enterprises give to the proposed contribution, as well as determining the practical relevance when applying the strategies alignment model in the $\mathrm{CN}$. For the validation of the proposed approach, a real simplified use case from food industry is presented. The simulated $\mathrm{CN}$ consists of two enterprises, the distributor $\left(E_{1}\right)$ and the manufacturer $\left(E_{2}\right)$, each one defining two objectives $\left(o_{i x k}\right)$ whose achievement is measured through the KPIs $\left(k p i_{i x k}\right): E_{1}$ defines $k p i_{111}$ and $k p i_{121} ; E_{2}$ defines $k p i_{211}$ and $k p i_{221}$. In order to achieve the objectives defined, each enterprise formulates two strategies $\left(s t r_{i s}\right)$ : $\mathrm{E}_{1}$ formulates

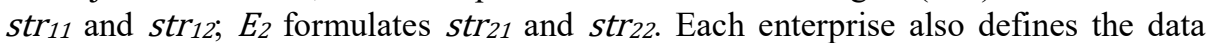
related to the strategies (durations and costs) and the associated to the corresponding KPIs (minimum values, threshold and weights). The objectives and the strategies are described in Section 3, in order not to repeat we refer the reader to that section. Moreover, the enterprises have a certain budget $\left(b_{i}\right)$ to carry on the formulated strategies. The values of influence that each strategy has on the defined KPIs are given by the parameter val_stris_kpi $k i_{x x k}$. All the data related with the objectives and strategies defined in the food industry use case are shown in Table 4. The data depicted on the cells in dark grey correspond to the values of influence that the strategies defined in one enterprise have on the KPIs defined in the same enterprise (intra-enterprise values of influence). While the white coloured cells represent the values related to the inter-enterprise influences. In the non-collaborative scenario only the inter-enterprise values of influence will be used. Whilst in the collaborative scenario will take into consideration both intra and inter-enterprise values of influence.

Table 4. Real simplified from food industry use case: Data

Distributor $\left(E_{\nu}\right)$
$\boldsymbol{b}_{l}=\mathbf{4}$

In the collaborative scenario the enterprises participating take into account the influences of all the strategies formulated by the enterprises. The optimisation experiment carried out in the simulation software used (AnyLogic) generates a set of solutions, as regards the units of strategies to activate and the time in which to activate them. The values concerning the enterprise performance indicators (kpi'i) and the network performance indicator (kpi'net) are computed in the simulation experiment. The experiments have been also performed in the non-collaborative scenario, in which the decision-making is made from an isolate perspective without considering how the strategies formulated by other network enterprises affect the achievement of its objectives (performance). In Table 5 the results of both scenarios, non-collaborative (NC) and collaborative (C) are compared. The optimised solution of 
the collaborative scenario (using the SAM) generates, at network level, a performance significantly higher than the performance resulting from the solution obtained in the non-collaborative scenario. Moreover, the solution obtained in the non-collaborative scenario breaches the restriction of non-negativity of all the KPIs of the network (fulfilment_kpi'ixk $>0$ ). Whereas that the solution of the collaborative scenario complies with the restriction of non-negativity being the fulfilment of all the KPIs 1.

Table 5. Collaborative scenario vs. Non-Collaborative scenarios: Optimization Results

\begin{tabular}{|c|c|c|c|}
\hline & $\mathrm{NC}$ & $\mathrm{C}$ & Performance Improvement \\
\hline$u_{-} s t r_{111}$ & 0 & 4,5 & \\
\hline ti_str ${ }_{111}$ & 0,105 & 0,013 & \\
\hline u_str ${ }_{121}$ & 1 & 0 & \\
\hline ti_str 121 & 0 & 0,017 & \\
\hline u_str 211 & 1 & 0 & \\
\hline ti_str 211 & 0,1 & 0,01 & \\
\hline$u_{-} s_{2} r_{221}$ & 0 & 2 & \\
\hline$t_{-} s_{t} r_{221}$ & 0,3 & 0 & \\
\hline$\nabla k p i_{11}^{\prime}$ & $-0,288$ & 0,674 & $334,03 \%$ \\
\hline fulfilment kpi' $_{111}$ & 0 & 1 & \\
\hline$\nabla k p i_{121}^{\prime}$ & 0,04 & 0,348 & $770,00 \%$ \\
\hline fulfilment kpi $^{\prime}{ }_{121}$ & 1 & 1 & \\
\hline$\nabla k p i_{211}^{\prime}$ & 0,226 & 0,951 & $320,80 \%$ \\
\hline fulfilment $\mathrm{kpi}^{\prime}{ }_{211}$ & 1 & 1 & \\
\hline$\nabla k p i_{221}^{\prime}$ & 0,141 & 1,307 & $826,95 \%$ \\
\hline fulfilment kpi' ${ }_{221}$ & 1 & 1 & \\
\hline$k p i_{1}^{\prime}$ (Distributor) & $-0,122$ & 0,511 & $518,85 \%$ \\
\hline kpi ${ }_{2}^{\prime}$ (Manufacturer) & 0,204 & 1,129 & $453,43 \%$ \\
\hline$k p i_{\text {net }}^{\prime}$ & 0,041 & 0,82 & $1900,00 \%$ \\
\hline
\end{tabular}

\section{Conclusions}

The developed research aims to provide a better understanding on the ways of establishing sustainable collaborative relationships within the partners of a CN. In this regard, a complete approach consisting of a model, a method a tool and a guideline is proposed, to support the strategies alignment process, in the $\mathrm{CN}$ context. The complete approach allows to automatically identifying the set of strategies to be activated, and the time in which to activate them, in order to obtain maximum levels of network performance. SD method is proposed to solve the SAM, and three tools support the computation of the SAM: (i) simulation software; (ii) DMS; and (iii) SAGEN tool, that automatically builds the SAM in the simulation software. Finally, a guideline is proposed, to give the $\mathrm{CN}$ partners a vision of how to perform the strategies alignment process. Despite the advantages of the application of the 
strategies alignment approach, there is a main drawback related with the information gathering as regards the value $v a l_{-} s t r_{i s_{-}} k p i^{\prime}{ }_{i x k}$, especially if the strategy $s t r_{i s}$ has never been activated before, this parameter it is very difficult to estimate. In the light of this, network enterprises can opt for (i) estimating the parameter $v a l_{-} s t r_{i s_{-}} k p i^{\prime}{ }_{i x k}$ or (ii) waiting until the strategy $\left(s t r_{i s}\right)$ is activated and measure the real value of $v a l \_s t r_{i s_{-}} k p i{ }_{i x k}$. If the enterprise has stored the increase of the KPIs when a strategy specific strategy was activated in the past $\left(\Delta k p i{ }_{i x k} \mid s t r_{i s}\right)$, the enterprise can objectively compute $v a l_{-} s t r_{j s_{-}} k p i^{\prime}{ }_{i x k}$, for strategies activated in the same enterprise; and $v a l_{-} s t r_{j s_{-}} k p i{ }_{i x k}$ for strategies active in different network enterprises.

Future research work leads to deal with the collection of the data required in an accurate way. For doing this, complementary sensitivity analysis is to be proposed in order to identify the robustness of the optimised solution obtained, resulting from the implementation of the SAM in the simulation software AnyLogic. A second future line of research leads to design a distributed multi-agent system model so that each network node is represented as an agent and simulates in its own hardware and software one part of the strategies alignment model (its own part). Moreover, other applications can be identified to the proposed work, such as supporting the partners' selection process from a collaborative perspective.

Acknowledgments. This work has been funded in part by Programa Val i+d para investigadores en formación (ACIF 2012).

\section{References}

1. R. Poler, L. M. Carneiro, T. Jasinski, M. Zolghadri, and P. Pedrazzoli, Intelligent Nonhierarchical Manufacturing Networks. Networks and Telecomunications Series. 2012. iSTE WILEY, 2012.

2. L. M. Camarinha-Matos and H. Afsarmanesh, Collaborative Networks: Reference Modelling. Springer International Publishing, 2008.

3. B. Andres and R. Poler, "Models, guidelines and tools for the integration of collaborative processes in non-hierarchical manufacturing networks: a review," Int. J. Comput. Integr. Manuf., vol. 2, no. 29, pp. 166-201, 2016.

4. B. Andres and R. Poler, "Relevant problems in collaborative processes of non-hierarchical manufacturing networks,” J. Ind. Eng. Manag., vol. 6, no. 3, pp. 723-731, 2013.

5. B. Andres and R. Poler, "Computing the Strategies Alignment in Collaborative Networks," in Enterprise Interoperability VI. Interoperability for Agility, Resilience and Plasticity of Collaborations, K. Mertins, F. Bénaben, R. Poler, and J.-P. Bourrières, Eds. Cham: Springer International Publishing, 2014, pp. 29-40.

6. U. Bititci, T. Turnera, D. Mackaya, D. Kearneyc, J. Parunga, and D. Waltersb, "Managing synergy in collaborative enterprises," Prod. Plan. Control Manag. Oper., vol. 18, no. 6, pp. 454-465, 2007.

7. P. Macedo, A. Abreu, and L. M. Camarinha-Matos, "A method to analyse the alignment of core values in collaborative networked organisations," Prod. Plan. Control, vol. 21, no. 2, pp. 145-159, 2010.

8. F. R. Piedade, A. Azevedo, and J. Bastos, "Managing Performance to Align the Participants of Collaborative Networks: Case Studies Results," in Collaborative Networks for 
Sustainable World, L. M. Camarinha-Matos, X. Boucher, and H. Afsarmanesh, Eds. Springer Berlin Heidelberg, 2010, pp. 545-552.

9. L. M. Camarinha-Matos and H. Afsarmanesh, "Collaborative networks: a new scientific discipline," J. Intelligent Manufacturing, vol. 16, No 4-5, pp439-452, 2005.

10. B. Andres and R. Poler, "A decision support system for the collaborative selection of strategies in enterprise networks," Decis. Support Syst., vol. 91, no. 10.1016/j.dss.2016.08.005, pp. 113-123, 2016.

11. Y. Kristianto, A. Gunasekaran, P. Helo, and M. Sandhu, "A decision support system for integrating manufacturing and product design into the reconfiguration of the supply chain networks," Decis. Support Syst., vol. 52, no. 4, pp. 790-801, 2012.

12. H. W. Lin, S. V. Nagalingam, S. S. Kuik, and T. Murata, "Design of a Global Decision Support System for a manufacturing SME: Towards participating in Collaborative Manufacturing,” Int. J. Prod. Econ., vol. 136, no. 1, pp. 1-12, 2012.

13. H. Seok, S. Y. Nof, and F. G. Filip, "Sustainability decision support system based on collaborative control theory," Annu. Rev. Control, vol. 36, no. 1, pp. 85-100, 2012.

14. F. Shafiei, D. Sundaram, and S. Piramuthu, "Multi-enterprise collaborative decision support system," Expert Syst. Appl., vol. 39, no. 9, pp. 7637-7651, 2012.

15. P. S. Tan, S. S. G. Lee, and A. E. S. Goh, "Multi-criteria decision techniques for contextaware B2B collaboration in supply chains," Decis. Support Syst., vol. 52, no. 4, pp. 779$789,2012$.

16. M. J. Verdecho, J. J. Alfaro, and R. Rodriguez, "Prioritization and management of interenterprise collaborative performance," Decis. Support Syst., vol. 53, no. 1, pp. 142-153, 2012.

17. T. P. Lu, A. J. C. Trappey, Y. K. Chen, and Y. D. Chang, "Collaborative design and analysis of supply chain network management key processes model," J. Netw. Comput. Appl., vol. 36, no. 6, pp. 1503-1511, 2013.

18. P. Macedo and L. M. Camarinha-Matos, "A qualitative approach to assess the alignment of Value Systems in collaborative enterprises networks," Comput. Ind. Eng., vol. 64, no. 1, pp. 412-424, 2013

19. R. K. Singh and L. Benyoucef, "A consensus based group decision making methodology for strategic selection problems of supply chain coordination," Eng. Appl. Artif. Intell., vol. 26, no. 1, pp. 122-134, 2013.

20. H. Zhang, Y. Deng, F. T. S. Chan, and X. Zhang, "A modified multi-criterion optimization genetic algorithm for order distribution in collaborative supply chain,” Appl. Math. Model., vol. 37, no. 14-15, pp. 7855-7864, 2013.

21. S. D. Dao, K. Abhary, and R. Marian, "Optimisation of partner selection and collaborative transportation scheduling in Virtual Enterprises using GA," Expert Syst. Appl., vol. 41, no. 15 , pp. 6701-6717, 2014.

22. R. Guillaume, G. Marques, C. Thierry, and D. Dubois, "Decision support with ill-known criteria in the collaborative supply chain context," Eng. Appl. Artif. Intell., vol. 36, pp. 1$11,2014$.

23. B. Andres, R. Poler, and R. Sanchis, "Collaborative Strategies Alignment to enhance the Collaborative Network agility and resilience,” IFIP Adv. Inf. Commun. Technol., vol. 463, pp. 88-99, 2015.

24. Lazarus Free Pascal, "Lazarus.” 2016.

25. B. Andres, R. Poler, J. Rosas, and L. Camarinha-matos, "Technological Innovation for Cyber-Physical Systems,” IFIP Int. Fed. Inf. Process., Springer, vol. 470, pp. 3-10, 2016.

26. J. P. C. Kleijnen and J. Wan, "Optimization of simulated systems: OptQuest and alternatives," Simul. Model. Pract. Theory, vol. 15, no. 3, pp. 354-362, 2007.

27. B. Andres and R. Poler, "Towards a Methodology to support the Strategies Alignment Process in Collaborative Networks," in Enterprise Interoperability VII. Enterprise Interoperability in the digitized and networked Factory of the Future, 2016, p. (In press). 
28. J. P. C. Kleijnen, "Verification and validation of simulation models," Eur. J. Oper. Res., vol. 82, pp. 145-162, 1995.

29. Akhras, G. (2000). Smart materials and smart systems for the future. Canadian Military Journal, 1(3), 25-31. 